\title{
REPRESENTAÇÕES SOCIAIS SOBRE ESOUECIMENTO E DEPRESSÃO POR PESSOAS IDOSAS: ABORDAGEM PROCESSUAL
}

Cristina Arreguy-Sena ${ }^{1}$

Amanda Maisa Gava Marangon Antônio Marcos Tosoli Gomes ${ }^{2}$ Laércio Deleon de Melo ${ }^{1}$

Renata Martins ${ }^{1}$
ORCID: http://orcid.org/0000-0002-5928-0495 ORCID: http://orcid.org/0000-0002-5120-2994 ORCID: http://orcid.org/0000-0003-4235-9647 ORCID: http://orcid.org/0000-0002-8470-7040 ORCID: http://orcid.org/0000-0003-0962-1885

Objetivo: compreender os conteúdos representacionais e suas origens a partir do esquecimento e da depressão em pessoas com idade $\geq 65$ anos, identificando-se as aproximações e as diferenciações atribuidas ao declínio cognitivo. Método: estudo qualitativo delineado na abordagem processual da Teoria das Representações Sociais, realizado numa Unidade de Atenção Primária à Saúde, numa cidade de Minas Gerais, Brasil. Entrevistas individuais em profundidade com gravação de áudio a partir de questões norteadoras. Conteúdos discursivos transcritos na íntegra e tratados em Programa NVivo Proll, análise de conteúdo. Atendidos todos os aspectos ético-legais de pesquisa. Resultados: foram 49 participantes, cujos fragmentos de discurso permitiram identificar dimensões: comportamental, atitudinal, cognitivo, informativo, valorativo e objetiva. A perda cognitiva foi percebida como algo peculiar ao envelhecimento e vinculada à presença da depressão. Conclusão: as representações sociais do esquecimento e depressão foram associadas ao isolamento social com origens vinculadas a problemas (pessoais, sociais ou familiares), identificadas a partir da observação do cotidiano.

Descritores: Enfermagem; Envelhecimento; Idoso; Memória; Depressão.

\section{SOCIAL REPRESENTATIONS OF FORGETTING AND DEPRESSION BY OLDER PEOPLE: PROCESS APPROACH}

Objective: to understand the representational contents and their origins from forgetfulness and depression in people aged $\geq 65$ years, identifying the approaches and differentiations attributed to cognitive decline. Method: qualitative study outlined in the procedural approach of the Theory of Social Representations, conducted in a Primary Care Unit. to Health in a city of Minas Gerais, Brazil. Individual in-depth interviews with audio recording from guiding questions. Discursive content transcribed in full and treated in NVivo Proll Program, content analysis. Met all ethical and legal aspects of research. Results: 49 participants, whose speech fragments allowed us to identify dimensions: behavioral, attitudinal, cognitive, informative, evaluative and objective. Cognitive loss was perceived as peculiar to aging and linked to the presence of depression. Conclusion: the social representations of forgetfulness and depression were associated with social isolation with origins linked to problems (personal, social or family) identified from the observation of daily life.

Descriptors: Nursing; Aging; Aged; Memory; Depression.

\section{REPRESENTACIONES SOCIALES DEL OLVIDO Y LA DEPRESIÓN DE LAS PERSONAS MAYORES: ENFOQUE DEL PROCESO}

Objectivo: comprender los contenidos de representación y sus orígenes del olvido y la depresión en personas de $\geq 65$ años, identificando los enfoques y las diferencias atribuidas al deterioro cognitivo. Metodo: estudio cualitativo descrito en el enfoque procesal de la Teoría de las Representaciones Sociales, realizado en una Unidad de Atención Primaria. a la salud en una ciudad de Minas Gerais, Brasil. Entrevistas individuales en profundidad con grabación de audio de preguntas orientadoras. Contenido discursivo transcrito en su totalidad y tratado en el programa NVivo Proll, análisis de contenido. Cumplió con todos los aspectos éticos y legales de la investigación. Resultados: 49 participantes, cuyos fragmentos de habla nos permitieron identificar dimensiones: conductuales, actitudinales, cognitivas, informativas, evaluativas y objetivas. La pérdida cognitiva se percibió como peculiar al envejecimiento y vinculada a la presencia de depresión. Conclusión: las representaciones sociales del olvido y la depresión se asociaron con el aislamiento social con orígenes vinculados a problemas (personales, sociales o familiares) identificados a partir de la observación de la vida cotidiana.

Descriptores: Enfermería; Envejecimiento; Anciano; Memoria; Depresión.

IUniversidade Federal de Juiz de Fora, MG

${ }^{2}$ Universidade Estadual do Rio de Janeiro,RJ.

Autor correspondente: Amanda Maísa Gava Marangon E-mail: amandamaisa@hotmail.com

Recebido: 02/07/2019

Aceito: 01/11/2019 


\section{INTRODUÇÃo}

Estima-se que o crescimento da população idosa mundial até 2050 aumente em 1,1 bilhão, tendo o número de idosos, na realidade brasileira, superado a 30 milhões em 2017. Entre as causas do envelhecimento populacional, destacam-se as influências do aumento da expectativa de vida, redução da taxa de fecundidade e das diferentes condições de vulnerabilidades e agravos à saúde ${ }^{(1,2)}$.

O envelhecimento é concebido além do critério cronológico, sendo um fenômeno natural, progressivo e irreversivel, que converge com modificações passiveis de serem explicadas por diferentes teorias ${ }^{(3)}$. Elas baseiam-se em manifestações clínicas conhecidas como síndromes geriátricas, ou seja, transformações que impactam a autonomia, independência e qualidade de vida do indivíduo, modificando o estado de cognição, humor, mobilidade e comunicação(4).

O humor, assim como a cognição, está ligado à perda da autonomia, podendo ser definido como a disposição, o ânimo para o desenvolvimento das funções e para a participação social. A cognição caracteriza-se pela capacidade mental de perceber e solucionar problemas do cotidiano. Ela envolve funções como: 1) o processo de memorização executado pelo córtex cerebral é responsável pelo armazenamento de informações; e 2) a conexão com outras áreas cerebrais capazes de assegurar a realização de ações complexas como o planejamento, monitoramento e o desempenho de atividades diárias, instrumentais e avançadas da vida que lhe favorecem a compreensão e engajamento no contexto social (2,3:5).

A possibilidade de ocorrência do declínio cognitivo pode resultar em alterações incapacitantes de memória, raciocínio, fluência fonética e semântica. Sua ocorrência pode ser caracterizada como: quedas ou ausências da identidade, expressas por perdas do raciocínio(4,5).

Com o declínio cognitivo, surge ainda a progressiva perda da capacidade funcional, instalando-se a dependência; esta por sua vez pode gerar ou agravar quadros depressivos, que justificam a demanda de atenção e cuidados na composição e disponibilidade da rede de apoio familiar e do sistema de saúde pública(6).

Seu impacto sobre a autonomia, a independência e a qualidade de vida, mediante a aproximação do processo de envelhecimento com os riscos do acometimento pelo esquecimento ou pela depressão(6), fazem com que um grupo de pessoas com idade $\geq 65$ anos possuam diferentes concepções, conhecimentos, informações, comportamentos, atitudes, imagens, valores e crenças a respeito do referido objeto(7). $^{(7)}$.

Partindo-se do pressuposto de que o grupo investigado convive em seu cotidiano de maneira (in)direta, com situações de esquecimento ou depressão em seu meio social, justifica-se a caracterização do mesmo como socialmente constituido(7). Eles justificam a idealização das representações do esquecimento e da depressão de pessoas com idade $\geq 65$ anos, como objeto de investigação. Para isso, optou-se pela abordagem processual da Teoria das Representações Sociais (TRS), como referencial teórico-metodológico(8)

Diante do exposto, objetivou-se compreender os conteúdos representacionais e suas origens a partir do esquecimento e da depressão em pessoas com idade $\geq 65$ anos, identificandose as aproximações e as diferenciações atribuidas ao declínio cognitivo.

\section{METODOLOGIA}

\section{Tipo de estudo}

Investigação qualitativa delineada na abordagem processual da TRS, segundo proposta de Moscovici(8).

\section{Participantes da pesquisa}

Amostra de conveniência com 49 moradores (idade $\geq 65$ anos) de uma região geográfica estimada em 200 idosos, cujo delineamento amostral envolveu: 1) amostra censitária de área física de moradores adstritos a uma Unidade Básica de Saúde (UBS), coincidente com região censitária do Instituto Brasileiro de Geografia e Estatística (IBGE) que compõem uma lista de pessoas integrantes das atividades de extensão universitária (2016-2019); e 2) subgrupo da amostra anterior definida por tipicidade.

Foram critérios de inclusão: pessoas com idade $\geq 65$ anos, adstritas a uma UBS, com nivel de orientação e capacidade comunicativa compativel com abordagem de entrevista e exclusão: pessoas não encontradas ou que adiaram a entrevista por $\geq 3$ agendamentos. Não houve perdas.

\section{Local do estudo}

O cenário da investigação foram os domicilios de idosos adstritos a uma UBS de uma cidade referência para ensino e serviços de saúde em uma macrorregião de Minas Gerais.

\section{Coleta de dados}

Instrumento de coleta de dados estruturado em: 1) determinantes sociodemográficos (gênero, idade, ano de escolaridade, ocupação, estado civil, cor da pele, $n^{\circ}$ filhos); 2) entrevista individual para captação de conteúdos discursivos e; 3) diário de campo.

O processo de coleta de dados ocorreu individualmente nos domicilios após agendamento prévio em horário compativel para a participante/pesquisadora. Houve treinamento prévio da pesquisadora para realizar entrevista em profundidade e captar as representações sociais. Coleta realizada entre novembro/2018 e janeiro/2019 (10-30 min). Assegurado anonimato dos participantes, com uso de códigos numéricos contendo cinco dígitos e privacidade da conversa durante a entrevista no ambiente familiar.

As variáveis de caracterização foram captadas no programa 
Open data Kit (ODK), visando reduzir o tempo de coleta de dados e evitar vieses na digitação. Os conteúdos discursivos gravados em áudio foram desencadeados pelas questões norteadoras: 1) "Conte-me um caso que tenha ocorrido com você ou que conheça a respeito da perda de memória" e 2) "Quais as características de uma pessoa com perda de memória e depressão?"

\section{Procedimentos de análise dos dados}

Os dados de caracterização dos participantes (variáveis qualitativas e quantitativas) foram tratados no programa Statistical Package for the Social Sciences (SPSS) versão 24 , por estatística descritiva, segundo análise de centralidade e dispersão (mediana, desvio-padrão, variabilidade).

Os conteúdos discursivos obtidos pela entrevista foram transcritos na integra, passando por leitura flutuante e aprofundada de todo o corpus com vistas à apropriação dos conteúdos e desencadeamento do processo de análise qualitativa de conteúdo que foi operacionalizado nas etapas: 1) pré-análise; 2) exploração do material e 3) resultados, inferência e interpretação(9). Utilizou-se como apoio o software NVivo Pro $11 \AA$, com categorias construídas a priori baseadas em dimensões e origens representacionais (adensamento teórico confirmado - correlação de Pearson $\geq 0,7$, representado em dendograma e gráfico de círculo).

\section{Procedimentos éticos}

Esta pesquisa integrou um estudo matriz (incapacidades cognitivas e atividades de vida diária em pessoas idosas: diagnóstico situacional), atendeu a todos os requisitos éticolegais de pesquisa em seres humanos (parecer consubstanciado $n^{\circ} 2.569 .508$ de 28/03/2018) com aquiescência dos participantes externada pela assinatura no Termo de Consentimento Livre e Esclarecido (TCLE) pós informado.

\section{RESULTADOS}

Participaram 49 pessoas com idade compreendida entre 65 a 99 anos (Md76,50 $\pm 58,057$ ), predominio de mulheres (71,4\%); cor de pele autodeclarada branca $(73,5 \%)$, tempo de estudo em anos compreendido entre 0-2l (Md4,00 $\pm 4,237$ ), casadas (51\%), com 0-12 filhos (Md3,00 $\pm 2,624$ ) e aposentadas (63,3\%).

A partir das dimensões representacionais e dos fragmentos que remetem à origem de tais representações, foi possivel identificar as categorias. 1) Esquecimento: marcador do declínio cognitivo (des)coberto no cotidiano; exemplificado pelo participante 65069 (Figura 1) e; 2) Depressão: alteração no engajamento social e no humor, (des)coberto na percepção de terceiros, conforme fala do participante $\mathbf{6 5 0 5 5}$ (Figura 1).

$\mathrm{Na}$ Figura 1, constam fragmentos de discursos exemplificando as origens e dimensões representacionais, conforme cada uma das categorias.

Figura 1 - Fragmentos de discursos sobre as dimensões, origens representacionais, dendograma e gráfico de círculo dos participantes conforme as categorias. Juiz de Fora, MG. Junho/2019.

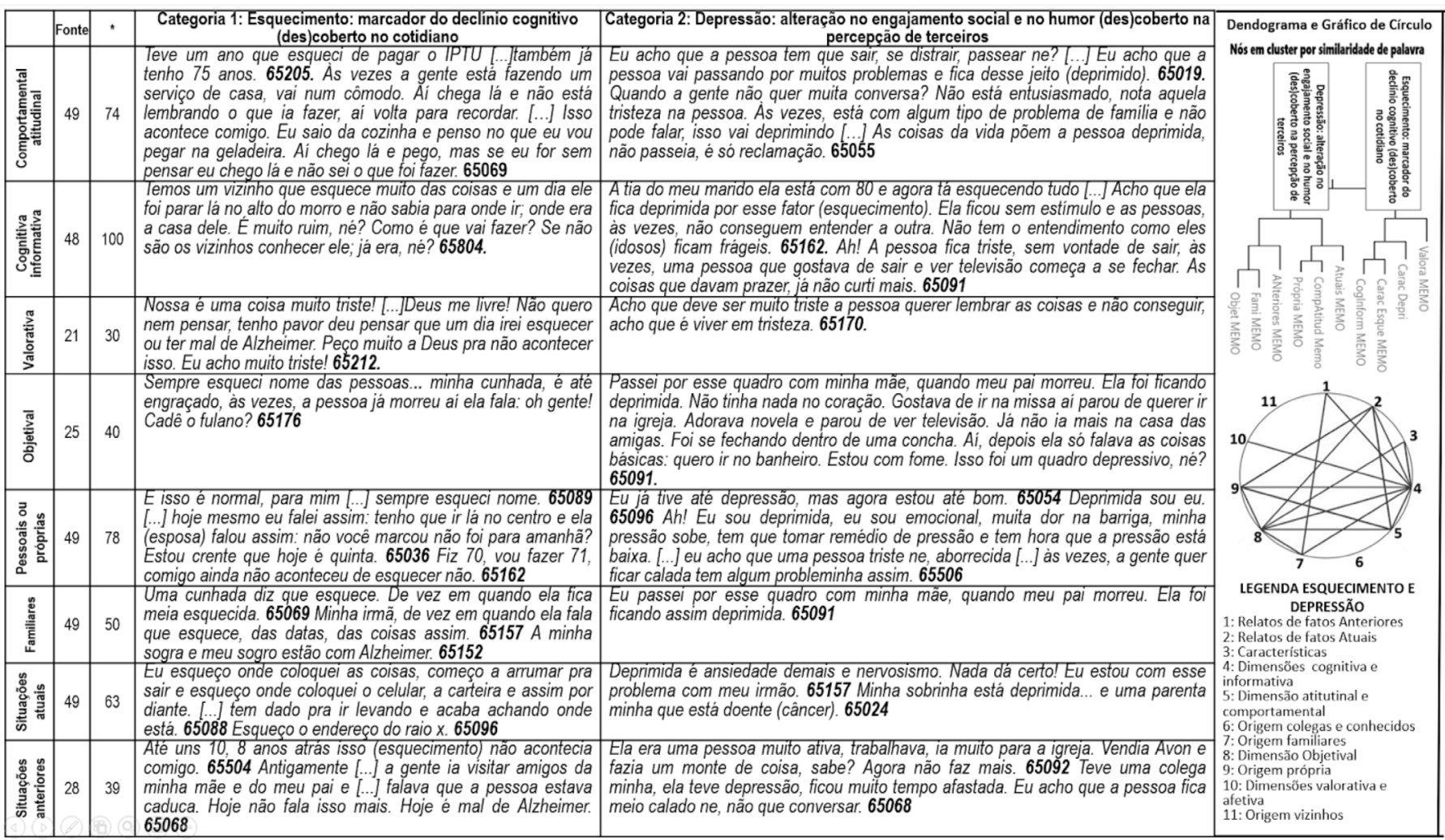

Fonte: NVivo Proll. *: $n^{\circ}$ fragmentos de discursos. 
A partir da análise do dendograma e do gráfico de círculo (Figura 1), compreende-se que o comportamento social do grupo está vinculado às memórias de suas experiências próprias ou com o cuidado de algum familiar idoso; as alterações cognitivas apresentadas descrevem as características habituais do esquecimento, que envolvem atividades cotidianas ou remotas ligadas ao esquecimento. Em contrapartida, a depressão está ancorada nas características sobre as doenças compartilhadas pelo grupo, de modo a descreverem o desengajamento e o isolamento social.

\section{DISCUSSÃO}

O perfil dos participantes, com predominância do sexo feminino $(71,4 \%)$, pode ser justificado pelo processo natural de feminilização do envelhecimento, visto que a expectativa de vida atual é de 79,8 anos para as mulheres, sendo 7,1 anos a mais do que para os homens ${ }^{(1)}$. A cor de pele declarada (73,5\% autodeclaram-se brancas) difere da equivalência de $46,7 \%$ caracterizados como parda, segundo estimativa brasileira. Porém, é necessário levar em consideração as variações regionais, justificadas pela miscigenação oriunda dos diferentes povos colonizadores, como os europeus ${ }^{(1,10)}$.

O número de filhos dos participantes e a identificação de $51 \%$ destes como casados corrobora a projeção de um núcleo familiar amplo como fator de proteção para as adaptações ao processo de envelhecimento e as limitações decorrentes das síndromes geriátricas, a exemplo do esquecimento e da depressão(11). A predominância de menos de cinco anos de escolaridade foi similar aos resultados de uma investigação com 180 pessoas, com idade $\geq 60$ anos, realizada em uma UBS do Paraná $(86,1 \%)^{(12)}$.

As reflexões a respeito das representações sociais do grupo foram discutidas na perspectiva das categorias elencadas, sendo a categoria "Esquecimento: marcador do declínio cognitivo (des)coberto no cotidiano", que elucida comportamentos e atitudes sobre esquecimento, que se apresentaram relacionados/ligados intimamente às atividades cotidianas.

O esquecimento, mesmo em pequenas proporções, esteve vinculado a não lembrança de nomes, pagamento de contas, localização de objetos e equipamentos assistivos. Para eles, o esquecimento é passivel de prevenção, mediante a estimulação da mente ou o engajamento em atividades como trabalhos manuais e ouvir música. Medidas de estimulação à cognição, apesar de estatisticamente, não demonstraram resultados significativos, com efeitos sobre a integração social, troca de experiências e empoderamento pessoal ${ }^{(13)}$.
Os conteúdos cognitivos/informativos, como dimensões do esquecimento, foram retratados a partir da convivência social com vizinhos, amigos ou familiares e aproximadas com os sintomas do Alzheimer, mesmo quando algumas pessoas apresentam manifestações distintas para a mesma doença. Os próprios participantes compreendem que as pessoas, por vezes, tratam mal ou sentem-se incomodadas com o esquecimento alheio e, com isso, a perda de memória os coloca à margem da sociedade. $\mathrm{O}$ isolamento decorrente dessa forma de apreensão do esquecimento esteve vinculado ao declínio cognitivo e à perda da juventude, os quais estavam ancorados em expressões de tristeza.

Entre os participantes, o valor negativo atribuído ao esquecimento foi justificado pela idade, porém, alguns se manifestaram conformados com essa perda de memória. Acreditavam tratar-se de algo inerente ao processo de envelhecimento, associando o esquecimento, a tristeza e o medo de que venham a ter esquecimento ou ser acometidos pelo Alzheimer. Os idosos vivem em constante processo de aceitação das alterações causadas pelo processo de envelhecimento, que precisam ser aceitas e subjetivas ${ }^{(14)}$.

Para exemplificar a dimensão objetiva, foram identificados substantivos próprios como o nome de familiares do núcleo cotidiano principal (ex. marido, filha). O esquecimento foi substanciado por meio do nome de pessoas e de atividades como compras, pelo fato de não lembrarem depois onde guardaram os objetos ou se os colocaram em lugares impróprios. O esquecimento foi considerado um evento cotidiano, marcador do declínio cognitivo, apesar de que sua ocorrência surgir ainda em pessoas jovens, mesmo que em menor proporção.

O declínio cognitivo tornou-se uma preocupação crescente para a população devido às suas implicações na diminuição da qualidade de vida, principalmente no que tange à independência, cuja perda é tão temida pelos idosos. Vale ressaltar que o envelhecimento cognitivo normal nada mais é do que mudanças no funcionamento do cérebro e não resulta necessariamente na demência; todavia, esses declínios na cognição podem afetar as habilidades funcionais e a independência ${ }^{(15)}$

Um estudo seccional verificou a associação entre capacidade cognitiva, condições socioeconômicas e estado nutricional,realizado com 180 idosos do Paraná, utilizando a escala de Mini-exame do Estado mental, um formulário para aferir as condições socioeconômicas e o índice de massa corporal. Identificou associação estatística entre capacidade cognitiva, sexo feminino (p-valor: 0,0442), capacidade cognitiva e escolaridade, compreendida entre 
l a 4 anos (p-valor: 0,0164), concluindo que o atendimento especializado aos idosos requer a identificação precoce dos problemas cognitivos e nutricionais ${ }^{(12)}$.

Na categoria: "Depressão: alteração no engajamento social e no humor (des)coberto na percepção de terceiros", a perda cognitiva como dimensão comportamental/ atitudinal esteve vinculada à depressão, à quantidade ou ao impacto dos problemas pessoais, sendo reconhecida sua presença por traços de tristeza, decorrentes da perda de pessoas significativas e contemporâneas. Os participantes reconheceram a necessidade de passear e se distrair como forma de prevenir a depressão.

Os conteúdos cognitivos e informativos acerca da depressão foram acessados a partir da perda de memória, justificada pela falta de estímulo e de entendimento da fragilidade, por aqueles com quem convivem. Os participantes caracterizaram as pessoas com depressão como alguém triste e que não tem mais prazer na realização de suas atividades de lazer. O lazer contribui para um melhor estado de espírito e, no caso dos mais idosos, diminui os sintomas depressivos e corrobora com a minimização dos sinais do processo de envelhecimento ${ }^{(16)}$

Os valores negativos atribuídos à depressão foram vinculados à perda de memória, uma vez que as pessoas se sentem envergonhadas por apresentarem algum episódio de esquecimento e se entristecem por não conseguirem acessar suas lembranças. Na concepção deles, a depressão favorece a marginalização e se manifesta através do isolamento social, alterações no humor e redução da capacidade de engajamento nas atividades grupais.

A depressão foi objetivada pelo grupo de modo a exemplificar características próprias da doença, a exemplo do desengajamento decorrente do isolamento social e do abandono de atividades antes consideradas prazerosas. Salienta-se que a depressão no idoso, frequentemente, surge num contexto de perda da qualidade de vida, associada ao isolamento social e ao surgimento de doenças graves $^{(17)}$

Cabe acrescentar evidências de seis grupos de fatores associados à depressão em idosos institucionalizados: sociodemográficos, condições de saúde, capacidade funcional, comportamento, cognição e medicamentos ${ }^{(16)}$, as quais podem ser analisadas e pensadas para um grupo de idosos não institucionalizados.

A partir da análise das representações do grupo, corroborada pelas informações do diário de campo, identificou-se que: a ancoragem ocorreu a partir das características próprias do esquecimento e da depressão, sendo consideradas possibilidades de ocorrência natural e próprias do agrisalhamento; a objetivação esteve circunscrita em acontecimentos e situações de vida atuais, ou anteriores, com informações compartilhadas pelo grupo, expressando o conhecimento social a respeito dos objetos investigados.

O grupo social considera o esquecimento e a depressão como passiveis de ocorrerem conforme vão envelhecendo e os identifica no seu cotidiano e convivência interpessoal por características de comprometimento cognitivo ou da depressão. Nesta perspectiva, evidências científicas recomendam que, em caso de coexistência de comprometimento cognitivo e/ou depressão identificada entre pessoas idosas, se deve analisar os biomarcadores de depressão tardia (LLD) e a doença de Alzheimer (DA) para diferenciá-los e direcionar adequadamente a pessoa para o tratamento e prognóstico ${ }^{(17)}$.

Limitações do estudo: considerando que o esquecimento e a depressão nem sempre são auto percebidos pela pessoa idosa, sugere-se a realização de investigações em outros grupos sociais, visando a captação de representações utilizando diferentes abordagens da TRS.

Contribuições do estudo: foram acessadas as representações sociais sobre o esquecimento e depressão, associadas ao processo de envelhecimento em um grupo de idosos que contribuem para subsidiar o cuidado de enfermagem, a partir da compreensão das respostas e necessidades identificadas no grupo em um contexto interdisciplinar e de visão ampliada de saúde.

\section{CONCLUSÃO}

As representações sociais sobre esquecimento e depressão foram objetivadas pelo isolamento social, com suas origens e dimensões ancoradas em situações pessoais, sociais ou familiares identificados no cotidiano. Por sua vez, a perda cognitiva é percebida como algo peculiar ao envelhecimento e ligada ao esquecimento, com possibilidade de ser vinculada à presença da depressão.

Contribuição dos autores: 1) concepção e/ou desenho: Arreguy-Sena e Marangon, 2) análise e interpretação dos dados: Tosoli, de Melo, Martins, Arreguy-Sena, Marangon e Silveira Fortes, 3) redação do artigo: Marangon, ArreguySena, Martins e de Melo, 4) revisão final: Marangon, Arreguy-Sena, Martins e de Melo. 


\section{REFERÊNCIAS}

1. Instituto Brasileiro de Geografia e Estatística. Projeções da população do Brasil e das Unidades da Federação [Internet]. Rio de Janeiro: IBGE; 2018 [cited 2019 Jul 1l]. Available from: https://www.ibge.gov.br/apps/populacao/projecao

2. Uchmanowicz I, Jankowska-Polańska B, Wleklik M, Lisiak M, Gobbens R. Frailty syndrome: nursing interventions. Sage Open Nurs [Internet]. 2018 [cited 2019 Jul 11]; 4(1):1-11. Available from: https://journals.sagepub.com/doi/ full/10.1177/2377960818759449

3. Simão LTSS, Lages LP, Paiva MHP, Ribeiro NLS, Araújo ERM, Leão GM. Perfil dos idosos com doenças crônicas não transmissiveis internados em unidade de terapia intensiva. Enferm. Foco [Internet]. 2019 [cited 2019 Jul 11]; 10(1):7680. Available from: http://revista.cofen.gov.br/index.php/ enfermagem/article/view/1329/499

4. Kim HJ, Min JY, Min KB. Successful aging and mortality risk: the Korean longitudinal study of aging (2006-2014). JAMDA [Internet]. 2019 [cited 2019 Jul 11]; 20(8):1013-20. Available from: https://linkinghub.elsevier.com/retrieve/ pii/S1525861018307175

5. Organização Mundial de Saúde. Resumo: Relatório mundial de envelhecimento e saúde [Internet]. Genève: WHO; 2015 [cited 2019 Jul 11]; 1-30. Available from: https://sbgg. org.br/wp-content/uploads/2015/10/OMS-ENVELHECIMENTO-2015-port.pdf

6. Bingham KS, Flint AJ, Mulsant BH. Management of late-life depression in the context of cognitive impairment: a review of recent literature. Curr Psychiatry Rep. 2019 [cited 2019 Jul 13]; 21(8):74. Available from: https://link.springer. com/article/10.1007/s11920-019-1047-7

7. Sá CP. Estudos de psicologia social: história, comportamento, representações e memória. 1 ed. Rio de Janeiro: EdUerj. 2015; 458p. ISBN: 978-85-7511-394-3.7.

8. Moscovici S. Representações Sociais: investigações em psicologia Social. 11 ed. Petrópolis: Vozes; 2015. 408p. ISBN: 8532628966

9. Bardin L. Análise de conteúdo. Trad. Luís Antero Reto e Augusto Pinheiro. Lisboa: Edições 70; 2011. 223p. ISBN: 9788562938047.

10. Brasil 2050 [recurso eletrônico] : desafios de uma na-
Ção que envelhece / Câmara dos Deputados, Centro de Estudos e Debates Estratégicos, Consultoria Legislativa; Brasilia: Edições Câmara, 2017 - (Série estudos estratégicos; n.8 PDF) [cited 2019 Jul 13]. ISBN 978-85-402-05772.

11. Cambuim AN, Almeida AB, Borrozzino NF, Lopes RGC. O conceito de familia na visão dos idosos. Rev Longeviver [Internet]. 2018 [cited 2019 Jul 13]; 9(57):4-8. Available from: http://www.portaldoenvelhecimento.com/index. php

12. Oliveira JM, Marçal DFS, Silva ES, Cortez LER, Bennemann RM. Cognição, condições socioeconômicas e estado nutricional de idosos cadastrados em uma unidade básica de saúde. Arq Ciênc Saúde. 2018 [cited 2019 Jul 13]; 25(2):3-7. Available from: http://www.cienciasdasaude.famerp.br/index.php/racs/article/view/795

13. Morando EMG, Schmitt JC, Ferreira MEC. Treino de memória em idosos saudáveis: uma revisão da literatura. Rev INFAD Psicol. 2018 [cited 2019 Jul 13]; 4(1): 293-310. Available from: http://www.infad.eu/RevistaINFAD/OJS/ index.php/IJODAEP/article/view/1136

14. Cherix K, Coelho Junior NE. Luto e melancolia nas demências: a psicanálise na clínica do envelhecimento. Trivium. 2018 [cited 2019 Jul 13]; 10(2):182-95. Available from: https://bdpi.usp.br/item/002916047

15. Hackett RA, Davies Kershaw H, Cadar D, Orrell M, Steptoe A. Walking speed, cognitive function, and dementia risk in the English longitudinal study of ageing. J Am Geriatr Soc. 2018 [cited 2019 Jul 13]; 66(9):1670-5. Available from: https://www.ncbi.nlm.nih.gov/pubmed/29508385

16. Ramos FP, Silva SC, Freitas DF, Gangussu LMB, Bicalho AH, Sousa BVO, et al. Fatores associados à depressão em idoso. Rev Eletr Acervo Saúde. 2019 [cited 2019 Jul 13]; 19(19):1-8. Available from: https://acervomais.com.br/index.php/saude/article/view/239

17. Liguori C, Pierantozzi M, Chiaravalloti A, Sancesario GM, Mercuri NB, Franchini F, et al. When cognitive decline and depression coexist in the elderly: CSF biomarkers analysis can differentiate alzheimer's disease from late-life depression. Front Aging Neurosci. 2018 [cited 2019 Jul 13]; 10:38. Available from: https://www.ncbi.nlm.nih.gov/pubmed/29527163 